\title{
Characteristics of blood pressure profiles and vascular dysfunction
}

\author{
Kenji Okumura and Xian Wu Cheng
}

Hypertension Research (2012) 35, 23-24; doi:10.1038/hr.2011.147; published online 1 September 2011

$\mathrm{H}$ ypertension is a well-known, important risk factor for the development of atherosclerosis and a strong predictor of cardiovascular morbidity and mortality. Although it has been more than half a century since blood pressure was first routinely assessed in the doctor's office, high blood pressure cannot, yet, be successfully prevented or controlled. ${ }^{1}$ The diagnosis and treatment of hypertension depends on correct measurement of blood pressure. However, this simple procedure is likely to involve several pitfalls, and the accuracy of blood pressure measurements is often reduced. In previous clinical studies, blood pressure measured in the doctor's office has been the standard, with multiple measurements permitting the calculation of an average for diagnosis and treatment. Previous epidemiological studies show that an average of office measurements proved to be an independent risk factor for high cardiovascular morbidity and mortality. Currently, data on various aspects of blood pressure, observed in various settings, have been collected for analysis. Home-based blood pressure measurement is able to identify so-called 'white-coat' hypertension and correlates with target-organ damage better than blood pressure values measured in the doctor's office. ${ }^{2} \mathrm{~A}$ number of studies using the evaluation of patterns of blood pressure variability over time have also been reported. ${ }^{3}$ At present, we can detect a variety of blood pressures under various conditions as well as blood pressure variability over time (Table 1 ); eventually, we would like to know which blood pressure measurement, or its variability, is the most important measure for predicting the

K Okumura is at the Department of Cardiology, The Toki Municipal General Hospital, 703-24 Tokiguchi, Tokitsucho, Toki, Gifu Prefecture 509-5193, Japan and XW Cheng is at the Department of Cardiology, Nagoya University Graduate School of Medicine, Nagoya, Japan. E-mail: kenji@med.nagoya-u.ac.jp development of atherosclerosis and the occurrence of cardiovascular events. Matsui et al. ${ }^{4}$ recently reported that the maximum home systolic blood pressure measured in the morning and evening for 14 consecutive days had the best predictive value for hypertensive target-organ damage, suggesting that atherosclerosis might be the most strongly associated with transient blood pressure fluctuations.

Blood pressure is always altered and significantly fluctuates from beat to beat in some cases, especially in patients with arrhythmia. Essentially, blood pressure is physiologically controlled by several systems based on neural receptors, such as baroreceptors, chemoreceptors and central nervous system ischemic response, and is easily shifted to another level within seconds (Figure 1). ${ }^{5}$ In addition, several hormonal and some minor systems modulate blood pressure within minutes. Finally, the kidney-fluid volume system affects blood pressure within hours or days. Guyton described how this latter factor contributes the most to controlling blood pressure after the arterial pressure suddenly becomes abnormal. ${ }^{6}$

In this issue of the Journal, Diaz et al. $^{7}$ estimated blood pressure, including visit-to visit and ambulatory blood pressure variability, office blood pressure and 24-hour blood pressure, in nonmedicated African Americans after a low-fat, low-salt diet and compared the values with flow-mediated vasodilation (FMD), nitroglycerin-mediated dilatation (NMD) and their ratio. They concluded that an increase in blood pressure variation, such as visit-to-visit systolic blood pressure variability, 24-hour systolic blood pressure variability and 24-hour diastolic blood pressure variability, is associated with reduced endothelial function as well as reduced vascular smooth muscle function. The assessment of FMD has been proposed to represent a functional bioassay for endothelium-derived nitric oxide (NO) bio- availability in humans and endothelial dysfunction is an early event in atherosclerosis. ${ }^{8}$ So far, extensive epidemiological evidence has consistently indicated that endothelial dysfunction of the coronary artery has a pivotal role in the development of atherosclerosis and cardiovascular disease. ${ }^{9}$ Similarly, it is possible that the assessment of endothelial function in the brachial artery may predict longterm cardiovascular risk, regardless of the method used for the assessment.

On the basis of the results obtained in this paper, Diaz et al. ${ }^{7}$ suggest that blood pressure variance is a more important predictor of cardiovascular diseases than office or home blood pressure, although their cross-sectional study shows only the relationship between vascular function and blood pressure variance and does not show a direct cause-and-effect relation between high blood pressure and cardiovascular events. Additionally, in this study, a single $0.4 \mathrm{mg}$ dose of a sublingual nitroglycerin tablet was given to assess NMD, an endothelium-independent vasodilation measure. Initially, NMD was used as a control test for FMD measurement to ensure that a reduced FMD capacity is a consequence of endothelial dysfunction, not a reflection

Table 1 A variety of blood pressure assessments

\begin{tabular}{ll}
\hline Aspect & Blood pressure assessment \\
\hline Setting & Office, ambulatory, home \\
Time & Daytime, nighttime, \\
& Morning, postprandial \\
Posture & Lying, sitting, standing \\
Variability & Reading-to-reading, \\
& Day-to-day, visit-to-visit \\
Instability & Office and home, \\
& Ambulatory monitoring \\
Reactivity & Physical tests, mental tests \\
\hline
\end{tabular}




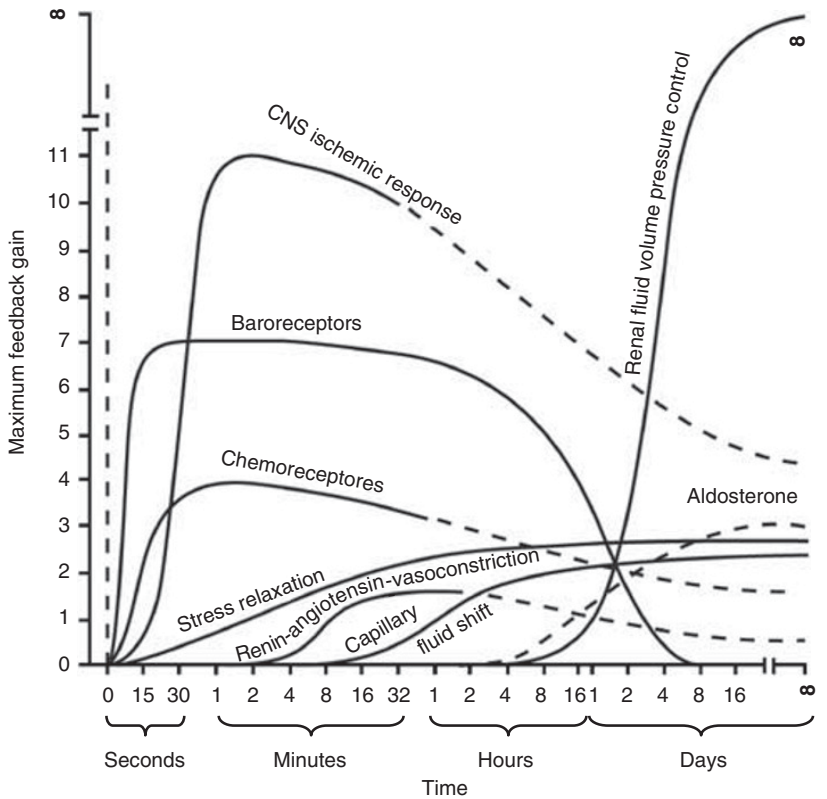

Figure 1 Degree of activation, expressed in terms of feedback gain at the optimal pressure, of different pressure control mechanisms after a sudden change in arterial pressure. (Redrawn from figure in the book by Guyton ${ }^{5}$.

of underlying smooth muscle dysfunction. ${ }^{10}$ This dose of nitroglycerin usually results in the maximum obtainable vasodilator response, ${ }^{11,12}$ which reflects vascular smooth muscle function. Currently, estimated brachial artery FMD is used to determine endothelial dysfunction, with impaired FMD thought to be an early sign of atherosclerosis. However, there is evidence that NMD, endotheliumindependent vasodilation, is also impaired in patients with atherosclerotic lesions, ${ }^{13}$ and that it poses a risk for the development of atherosclerosis ${ }^{14}$ in addition to influencing endothelial function because the relaxant response of smooth muscles to an exogenous source of NO is impaired in vessels, even at an early stage of atherosclerosis. In normotensive children with diabetes, impaired NMD is associated with reduced FMD, increased oxidative stress and preclinical carotid atherosclerosis..$^{15}$ NMD should, therefore, receive due attention when evaluating changes in vascular responsiveness.

Because the level of FMD is dependent on the strength of the shear stress from blood flow, it is reasonable to normalize FMD by dividing the percentage of FMD by the shear rate. ${ }^{16}$ However, it is not easy to determine the accurate shear stress. The ratio of FMD (\%) to NMD (\%) (FMD/NMD) also seems to be an alternative and better method for normalization of FMD, as presented in this study, because the arterial response induced by intrinsic NO is related to vascular smooth function. ${ }^{17}$ Moreover, FMD appears to also be affected by the basal brachial artery diameter, which is related to the arterial responsiveness because of changes in the structural and functional properties of the vasculature according to artery diameter. The difference in the responsiveness by conduit artery diameter could be normalized by the index FMD/NMD. Unfortunately, only a few studies have analyzed the FMD/NMD ratio. In this paper, the authors proposed that the FMD/NMD ratio is the best available marker of endothelial function on the basis of their results and the results of others. ${ }^{10,17,18}$

Blood pressure is easily measured anytime under various conditions, and the estimation of blood pressure variability is currently also easily obtainable, but the reliability of measured blood pressure levels is still low. Differences in blood pressure assessments should receive more attention so that clinicians can take into account differences in measurement procedures and environmental conditions. An approach such as that described by Diaz et al. ${ }^{7}$ using ambulatory blood pressure monitoring can provide information on which is the best assessment of blood pressure, allowing researchers to refine the risk stratification for cardiovascular disease in patients with hypertension. The results obtained in these studies are sure to be reflected in future guidelines for the management of hypertension.

\section{CONFLICT OF INTEREST}

The authors declare no conflict of interest.
1 Messerli FH, Williams B, Ritz E. Essential hypertension. Lancet 2007; 370: 591-603

2 Mancia G, Zanchetti A, Agabiti-Rosei E, Benemio G, De Cesaris R, Fogari R, Pessina A, Porcellati C, Rappelli A, Salvetti A, Trimarco B. Ambulatory blood pressure is superior to clinic blood pressure in predicting treatment-induced regression of left ventricular hypertrophy. Circulation 1997; 95: 1464-1470.

3 Hansen TW, Thijs L, Li Y, Boggia J, Kikuya M, Björklund-Bodegård K, Richart T, Ohkubo T, Jeppesen J, Torp-Pedersen C, Dolan E, Kuznetsova T, Stolarz-Skrzypek K, Tikhonoff V, Malyutina S, Casiglia E, Nikitin Y, Lind L, Sandoya E, Kawecka-Jaszcz K, Imai Y, Wang J, Ibsen $\mathrm{H}$, O'Brien E, Staessen JA. Prognostic value of reading-to-reading blood pressure variability over 24 hours in 8938 subjects from 11 populations. Hypertension 2010; 55: 1049-1057.

4 Matsui Y, Ishikawa J, Eguchi K, Shibasaki S, Shimada K, Kario K. Maximum value of home blood pressure: a novel indicator of target organ damage in hypertension. Hypertension 2011; 57: 1087-1093.

5 Guyton AC. Circulatory Physiology III: Arterial Pressure and Hypertension. Philadelphia, PA: WB Saunders, 1980.

6 Guyton AC. Blood pressure control: Special role of the kidneys and body fluids. Science 1991; 252: 1813-1826.

7 Diaz KM, Veerabhadrappa P, Kashem MA, Feairheller DL, Sturgeon KM, Williamson ST, Crabbe DL, Brown MD. Relationship of visit-to-visit and ambulatory blood pressure variability to vascular function in African Americans. Hypertens Res 2012; 35: 55-61.

8 Green D. Point: flow-mediated dilation does reflect nitric oxide-mediated endothelial function. J App/ Physiol 2005; 99: 1233-1234.

9 Suwaidi JA, Hamasaki S, Higano ST, Nishimura RA, Holmes Jr DR, Lerman A. Long-term follow-up of patients with mild coronary artery disease and endothelial dysfunction. Circulation 2000; 101: 948-954.

10 Celermajer DS, Sorensen KE, Bull C, Robinson J, Deanfield JE. Endothelium-dependent dilation in the systemic arteries of asymptomatic subjects relates to coronary risk factors and their interaction. J Am Coll Cardiol 1994; 24: 1468-1474.

11 Inoue T, Matsuoka H, Higashi Y, Ueda S, Sata M, Shimada KE, Ishibashi Y, Node K. Flow-mediated vasodilation as a diagnostic modality for vascular failure. Hypertens Res 2008; 31: 2105-2113.

12 Ducharme A, Dupuis J, McNicoll S, Harel F, Tardif JC. Comparison of nitroglycerin lingual spray and sublingual tablet on time of onset and duration of brachial artery vasodilation in normal subjects. Am J Cardiol 1999; 84: 952-954.

13 Raitakari OT, Seale JP, Celermajer DS. Impaired vascular responses to nitroglycerin in subjects with coronary atherosclerosis. Am J Cardiol 2001; 87: 217-219.

14 Adams MR, Robinson J, McCredie R, Seale JP, Sorensen KE, Deanfield JE, Celermajer DS. Smooth muscle dysfunction occurs independently of impaired endothelium-dependent dilation in adults at risk of atherosclerosis. J Am Coll Cardiol 1998; 32: 123-127.

15 Järvisalo MJ, Lehtimäki T, Raitakari OT. Determinants of arterial nitrate-mediated dilatation in children: role of oxidized low-density lipoprotein, endothelial function, and carotid intima-media thickness. Circulation 2004; 109: 2885-2889.

16 Harris RA, Nishiyama SK, Wray DW, Richardson RS. Ultrasound assessment of flow-mediated dilation. Hypertension 2010; 55: 1075-1085.

17 Chan SY, Mancini GB, Kuramoto L, Schulzer M, Frohlich J, Ignaszewski A. The prognostic importance of endothelial dysfunction and carotid atheroma burden in patients with coronary artery disease. J Am Coll Cardiol 2003; 42: 1037-1043.

18 Imamura A, Takahashi R, Murakami R, Kataoka H, Cheng XW, Numaguchi $\mathrm{Y}$, Murohara T, Okumura K. The effects of endothelial nitric oxide synthase gene polymorphisms on endothelial function and metabolic risk factors in healthy subjects: the significance of plasma adiponectin levels. Eur J Endocrinol 2008; 158: 189-195. 